(2) Comparing the groups of pilots of long experience and distinction with the accepted candidates, a diminution in the frequency of visual defects amounting to 4 per cent. of the total numbers examined is apparent in the former.

(3) Three quarters of this diminution has occurred in the larger, and one quarter among the smaller defects.

(4) Of 383 experienced pilots, 93, who in the aggregate had flown for 75,000 hours, failed to reach the standard of normal vision in each eye. Of these, 65 had small and 8 large defects within the standard fixed, while 20 had defects ranging from $6 / 12,6 / 18$ as high as $6 / 60$ in each eye.

\title{
THE RADICAL CURE OF GONORRHOEAL IRITIS.*
} BY

\author{
S. H. BRowning, M.R.C.S., L.R.C.P., \\ BACTERIOLOGIST TO THE ROYAL LONDON OPHTHALMIC HOSPITAL AND \\ ALL SAINTS' HOSPITAL FOR GENITO-URINARY DISEASES.
}

GONORRHOEAL iritis being a late sequel of gonorrhoea, is not as a rule seen first hand by a specialist in venereal diseases, with the result that it is more often than not treated as a local affection. In this way it has come to be talked of as " recurrent iritis," whereas few if any cases of gonorrhoeal iritis ought to occur in the first place, and certainly ought not to recur if properly treated.

The object of this paper is to try to show that by proper treatment of the genito-urinary tract and the cure of the disease existing there, a permanent cure of the gonorrhoeal iritis will follow; also to bring to the notice of ophthalmic surgeons their responsibility in seeing that their cases of gonorrhoeal iritis are adequately treated in the way I shall indicate. Gonorrhoeal iritis is, I think, a toxic condition and is not due to the presence of the gonococcus in the eye, for this organism has only been isolated from the eye in one case (Sidler-Huguenin), and then only from the blood stained exudate from the anterior chamber of a patient suffering from acute gonorrhoeal septicaemia. I have examined the gelatinous exudate from cases of gonorrhoeal iritis under the care of Mr. Lang, but have not been able to cultivate the gonococcus or find the organism in direct smears. These experiments were carried out under the most favourable conditions as the exudate was in all cases planted out on to suitable media within a few seconds of being drawn off.

Then, again, the rapid cures I have sometimes had after vaccine

\footnotetext{
${ }^{*}$ Read in the Section of Ophthalmology of the Royal Society of Medicine on Feb. 4, 1920.
} 
treatment more closely resemble recovery from a toxic condition than a microbic one.

That the iritis is gonorrhoeal is suggested by the following observations :

1. There is generally a definite history of gonorrhoea some years previously. I may here mention that I have never seen a case of iritis during the acute stage of an urethritis, which observation is confirmed by Mr. Canny Ryall, of All Saints' Hospital, and also by Mr. C. H. Mills, who has been a specialist at the Military Hospital, Rochester Row, for the last five years. The observations of these two surgeons must run into many thousands of cases.

2. The specific reaction to gonorrhoeal vaccines.

3. Its association with gonorrhoeal rheumatism, though this is by no means invariably the case.

4. The recurrence of iritis or exacerbation of symptoms after prostatic and vesicular massage, probably due to the liberation of toxins and bacteria - dead or alive-into the blood stream.

5. By negative evidence; that is to say, by eliminating as far as possible all other sources of infection such as pyorrhoea, septic tonsils and ears, cystitis, alimentary infections, etc.

I have not mentioned the complement fixation test in the above list, as I did not do it in any of the cases I am about to record, and also because in my hands it has not yet proved reliable and I do not yet know of a method which gives consistently true results.

Treatment.-Treatment must be considered under two headings, preventive and radical.

The preventive treatment of gonorrhoeal iritis really rests with those who see the gonorrhoea in its early stages, as no case of gonorrhoea can be said to be cured till the prostate and vesicles have been carefully examined, and proved by repeated examination of their contents, expressed by massage, to be free from infection. Examination with the urethroscope should also be carried out. I believe that if the above examinations were done by a specialist in genito-urinary work and a bacteriologist familiar with the examination of urines after massage, there would be no such eye condition as "gonorrhoeal" iritis.

I think that it is now generally recognized that all cases of gonorrhoea should be treated with vaccines, for although they do not materially shorten the acute stage of the disease, there is considerable evidence to show that complications and sequelae are to a large extent prevented by their use. The radical treatment of gonorrhœal iritis I would suggest to be as follows:

1. The immediate treatment by the ophthalmic surgeon.

2. The immediate treatment by vaccines.

3. Continued treatment by vaccines and prostatic and vesicular massage with treatment of the urethra if necessary. 
1. Of the immediate and continued treatment by the ophthalmic surgeon I of course can have nothing to say, but the responsibility generally rests with him as to whether the subsequent treatment is advised or not.

2. I am convinced that the immediate treatment by gonococcal vaccines is essential if the best is to be done for the patient. The acute attack is often cut short and the relief to the patient as regards pain is definite.

An autogenous vaccine should be used if possible, but while this is being prepared suitable doses of a reliable mixed vaccine may be given.

Vaccine therapy has rather gone out of fashion and repute the last few years, and this I think, is due to the haphazard use of commercial vaccines by persons unskilled in vaccine therapy. Vaccines freshly prepared from many strains are necessary for stock use, and this is seldom obtained in those bought in a chemist's shop. However, I have found the French preparation called "Dmégon" and Mulford's vaccine to be really good and reliable.

The dose of vaccine varies with each patient and I have used initial doses of from 5 to 500 millions with success. I would here remind you that vaccine therapy is an aid to general medical and surgical treatment and is in no way intended as a substitute.

3. I now come to an important part of the treatment and that is the examination of the genito-urinary tract. Practically every case of gonorrhoeal iritis I have examined or have had examined, has shown some disease of the prostate or vesicles, and massage of these organs has shown the presence of pus cells and often gonococci. The cure of the iritis depends or the cure of the accompanying prostatitis or vesiculitis and this is generally brought about by massage.

Massage of the prostate and vesicles is a difficult and arduous operation and unless done by an expert is useless, as much judgment must be used as to how much or how little massage any one prostate will stand. Only those thoroughly familiar with the normal and diseased organs can carry out the treatment successfully.

The method usually adopted is as follows:

The patient is told to come with a full bladder, and before massage a little urine is passed into a specimen glass in order to test for threads, pus, or micro-organisms. He then gets up on to a couch or table in the knee-elbow position and the well lubricated forefinger is passed into the rectum. The prostate is then massaged with a sweeping motion round the edges of the lobes, first on one side and then on the other, firm pressure being applied all the time and working towards the centre; this is continued for two or three minutes. The finger is then passed over the prostate and the vesicles are massaged and emptied of their contents, pressure is 
applied to the abdomen with the left hand at the same time in order to push the bladder back and thus enable the vesicles to be more easily reached. This is an important point.

The patient then passes his water into a glass and the centrifugalized deposit is examined for pus, gonococci or other organisms.

Gonococci are not found in all cases of iritis, but this is probably due to the difficulty of detecting the organism which is often swollen and stained badly, or is lost in a mass of spermatozoa, pus cells, epithelium, and other organisms, but the fact remains that even when the gonococcus is not found the iritis is cured when the urine examined after massage repeatedly gives a normal desposit.

I do not think that iritis is often due to the other bacteria found after massage, such as staphylococci, B.coli, diphtheroids, etc., though Mr. Canny Ryall has told me of a case of iritis in which a pure culture of the bacillus coli was isolated from the urine after massage and which was cured without recurrence after suitable treatment. I have not yet seen a case of gonorrhoeal iritis in a female. The following is a list of some of the cases I have treated or had treated by Mr. Canny Ryall or by Mr. C. H. Mills and my thanks are due to them for the great trouble and interest they have taken with the cases, also to Mr. Lang, Mr. Worth, Mr. Hudson, Mr. Greeves, and Mr. Whiting for permission to publish their cases.

I have followed up these cases as far as possible, and with one exception there has not been a recurrence for at least the last five years.

The recurrence was in a case of Mr. Worth's, and he came to see me last month with an acute iritis and begged for vaccine treatment and a letter to All Saints' Hospital. He had been to this hospital before, but for certain reasons had been unable to continue treatment, although he was told he was not cured. He had been four and a half years without a recurrence. Mr. Ryall saw the case and demonstrated the enlarged prostate and matted vesicles to be felt in the rectum. Examination of the urine after massage showed the presence of many pus cells and a considerable number of gonococci. He is now undergoing a thorough course of massage and vaccine treatment.

CASE 1. A., married. History of gonorrhoea 18 years ago; slight rheumatism at times, recurrent iritis for last six years; left iridectomy, massage, ànd vaccines, cured.

CASE 2. B. History of gonorrhoea six years ago, sixth attack; massage and vaccines; cured.

CASE 3. C. Many attacks of recurrences of gonorrhoea, often with rheumatism; cannot remember the date of first attack. His wife has chronic "tubes"; no children. A difficult case, which developed another attack during treatment, but eventually cleared up completely and there has been no recurrence for the last five years. 
CASE 4. D. History of gonorrhoea five years ago; no rheumatism ; two attacks of iritis; cured.

CASE 5. E. History of gonorrhoea six or seven years ago; cured.

CASE 6. F. History ten years; did not improve much with vaccine treatment alone, but got quite well with massage and vaccines combined.

CASE 7. Mentioned above.

CASE 8. O. R. History of gonorrhoea 17 years ago ; first attack of iritis ; massage and vaccines ; cured.

CASE 9. M. J. History two years; third attack of iritis in five months ; cured.

CASE 10. I. M. S. History of many attacks of gonorrhoea with acute epididymitis and prostatitis; one eye practically blind as the result of many attacks of iritis, cured.

Gonococci were found in the material obtained after massage in all the above cases.

\title{
THE ACTION OF HYPOPHYSIN (PITUITRIN) UPON THE PUPIL OF THE RABBIT* \\ BY
}

\author{
W. B. Inglis Pollock \\ GLASGOW.
}

\section{Introduction}

THE problem of the determination of the precise seat of action of drugs has proved one of the most difficult in the whole range of pharmacology. Especially is this the case as regards their action on visceral muscular structures... While, in the skeletal muscles, a definite fibre extends from a cell in the central nervous system to one or more muscle fibres where it ends in definite end-plates, in visceral muscles the fibre is interrupted at least once in its course, and this interruption may occur almost anywhere between the spinal cord and the muscles supplied. It is generally assumed that there is but one synapse, one cell station in each fibre, but the evidence for this is far from conclusive.

Probably the most satisfactory evidence is that aftorded by the action of nicotin on the heart of the frog. The vagus endings are paralysed, but the augmentor nerves still act. Hence the conclusion is arrived at that the former have their cell station in the-heart, and the latter in the stellate ganglion. But while such an experiment indicates that the greater proportion of synapses of the former are

* From the Department of Physiology, University of Glasgow. 